\title{
Implantation of a Permanent Pacemaker in a Patient With Severe Parkinson's Disease and a Preexisting Bilateral Deep Brain Stimulator
}

\author{
Beste OzBen, ${ }^{1} \mathrm{MD}$, Ahmet Kaya BILGE, ${ }^{1} \mathrm{MD}$, Ercument Yilmaz, ${ }^{1} \mathrm{MD}$, \\ and Kamil ADALET, ${ }^{1} \mathrm{MD}$
}

\begin{abstract}
SUMMARY
Cardiac pacemakers and implantable defibrillators are commonly used therapeutic modalities in cardiac arrhythmias. Thalamic deep brain stimulation has also become an important modality in the treatment of drug-refractory tremors and other complications in advanced Parkinson's disease. Concerns exist about the potential electrical interaction and interference between these 2 devices in the same patient. There are only a limited number of reports that have investigated this issue. We describe a patient with advanced Parkinson's disease and a previously implanted deep brain stimulator, who subsequently needed a permanent cardiac pacemaker due to severe bradyarrhythmia. Despite the probability of interference between the devices, there were no problems during implantation of the cardiac pacemaker; both the deep brain stimulator and cardiac pacemaker functioned appropriately afterwards. (Int Heart J 2006; 47: 803-810)
\end{abstract}

Key words: Parkinson's disease, Deep brain stimulation, Cardiac pacemaker

IN the past decade, medical science has witnessed the increasing use of implantable chips and biosensors. Implantable cardiac pacemakers and defibrillators have for many years been commonly used therapeutic modalities for certain cardiac arrhythmias or dysrhythmias. ${ }^{1)}$ Also, continuous high frequency thalamic deep brain stimulation (DBS) is being used for the treatment of selected patients with disabling, drug-refractory essential tremor or advanced Parkinson's disease. ${ }^{2)}$ Concerns exist about the potential electrical interaction and interference between these 2 devices (or with device programming) in the same patient. Thus, potential operational safety and efficacy issues must be defined. There are limited numbers of reports about this issue. While deep brain stimulators and cardiac pacemakers/implantable cardioverter defibrillators (ICD) have potential risks for operative and postoperative complications, these modalities are usually associated with minimal long-term complications. ${ }^{2-4)}$ However, electrical devices,

From the ${ }^{1}$ Department of Cardiology, Istanbul Faculty of Medicine, Istanbul University, Istanbul, Turkey.

Address for correspondence: Kamil Adalet, MD, Cardiology, Yildiz Caddesi Konak Apartmani, No: 43/24, 34353, Besiktas, Istanbul, Turkey.

Received for publication November 17, 2005.

Revised and accepted July 6, 2006. 
including deep brain stimulation pulse generators or pacemakers, may mutually interfere, and device-device or device-programmer interactions may occur. We describe a patient who underwent successful thalamic deep brain stimulation for essential tremor due to advanced Parkinson's disease, who later developed cardiac bradyarrhythmia requiring a pacemaker.

\section{Case Report}

A 41-year-old patient was referred to our cardiology department with a diagnosis of dilated cardiomyopathy and severe left ventricular systolic dysfunction. Parkinson's disease was diagnosed in 1995 and he was being followed by the neurology department. Despite optimal drug therapy, he had disabling, medically refractory tremor and speech disturbances. During routine follow-up in neurology clinics, due to being refractory to medical treatment and the advanced state of the Parkinson's disease, implantation of a deep brain stimulator (Medtronic) was undertaken in Germany in 2002. He had been complaining of dyspnea and orthopnea in 2003. Echocardiographic examination revealed severe left ventricular systolic dysfunction (with an ejection fraction of $25 \%$ ) with global hypokinesia of the left ventricular walls, dilatation of the left chambers, and degenerative aortic and mitral valves with moderate mitral regurgitation. His electrocardiogram revealed atrial fibrillation with a very slow ventricular conduction. He was hospitalized and standard medical treatment (angiotensin converting enzyme inhibitor, furosemide, spironolactone) was given for congestive heart failure. His family history was interesting in that his 2 elder brothers had also cardiomyopathy and suffered from sudden death.

Nonsustained ventricular tachycardia episodes and pauses greater than 2 seconds were detected on his 24-hour ambulatory ECG recording. Electrophysiology and coronary angiography were planned and he was told that he might require a cardioverter defibrillator. However, the patient refused all further procedures and was discharged with medical treatment.

Six months later, he was hospitalized due to a left posterior cerebral artery occlusion-related infarction. During hospitalization, he had dyspnea and was reminded again of the need for cardiac intervention. He agreed to further cardiac examinations and was referred from the neurology department to our cardiology department. He was admitted to the intensive care unit of the arrhythmia division. Physical examination revealed a blood pressure of 100/55 $\mathrm{mmHg}$ and heart rate of 55/min. Cardiac examination showed normal first and second heart sounds with a pansystolic murmur in the mitral area. His lungs were clear to auscultation and there was no hepatosplenomegaly. A neurological examination revealed disartria, bilateral rigidity, and bradykinesia (more prominent on the right side of the 


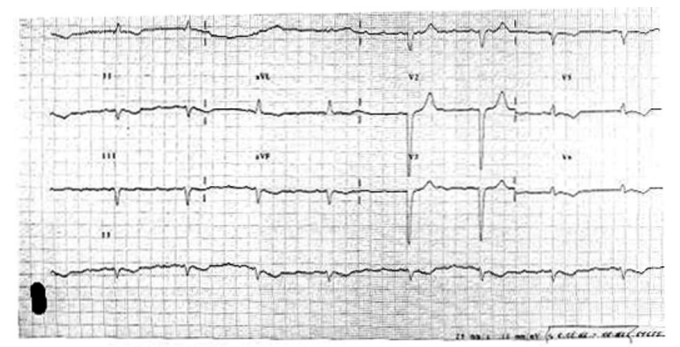

Figure 1. ECG showing atrial fibrillation with a slow ventricular response.

A

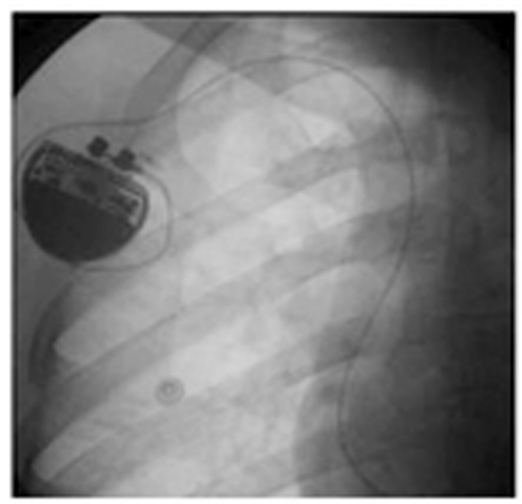

B

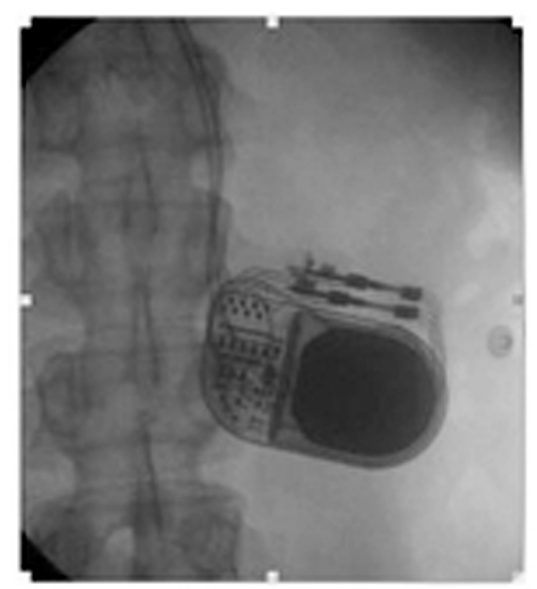

Figure 2. Pacemaker generator in right subclavicular area (A) and deep brain stimulator generator (B) in left upper abdomen.

patient). In the ECG, atrial fibrillation was present with a slow ventricular response and incomplete left bundle branch block (Figure 1). An echocardiographic examination showed severe dilatation of the left ventricle (diastolic diameter: $6.30 \mathrm{~cm}$, systolic diameter: $5.60 \mathrm{~cm}$ ) and global hypokinesia with severe systolic dysfunction (left ventricular ejection fraction was $26 \%$ via arealength method). There were degenerative changes in the aortic and mitral valves with mild to moderate mitral regurgitation. An apical organized thrombus was also detected. A hemogram and biochemical analysis were normal.

Enoxiparin sodium $(2 \times 40 \mathrm{mg} / \mathrm{day})$, ramipril/hydrochlorothiazide $(1 \times 5 / 25$ $\mathrm{mg} /$ day $)$, spironolactone $(1 \times 25 \mathrm{mg} /$ day $)$, levodopa/benserazide $(4 \times 150 / 37.5$ $\mathrm{mg} /$ day $)$, entacapone $(4 \times 200 \mathrm{mg} /$ day $)$ and pramipexole $(3 \times 0.125 \mathrm{mg} /$ day $)$ were administered. During continuous cardiac rhythm monitoring, atrial fibrillation episodes with high atrioventricular block leading to a ventricular rate of 20-25/ 
min and nonsustained tachycardia episodes with broad QRS were seen. Coronary angiography revealed normal coronary arteries. Sustained ventricular arrhythmia was not induced in electrophysiological examination. Implantation of a single chambered pacemaker and bipolar leads was performed. During implantation of the VVI-R pacemaker (Insignia I Entra 1198, Guidant), the deep brain stimulator was first turned off. The pacemaker was implanted in the right subclavicular area, on the opposite side to the deep brain stimulator (Figures 2A and 2B). After the lead was positioned into the right ventricle, the deep brain stimulator was turned on and repetitive testing was undertaken to ensure that no device-device interaction occurred. The intracardiac electrocardiogram was observed while the rate, voltage, and pulse width of the deep brain stimulator generator were increased to maximum levels ('worst case scenario'). No inappropriate sensing was evident through the lead (Figure 3). After the pacemaker was connected to the ventricular lead, testing was repeated with no evidence of inappropriate sensing through the device. The ventricular sensing was $2 \mathrm{mV}$ and the output setting of brain stimulus was $4 \mathrm{mV}$. The patient tolerated the procedure well and had no complications. During hospitalization, no sign of interference was observed on the 24-hour rhythm monitoring and an ECG showed regular pacemaker rhythm (Figure 4).

At 6-month follow-up, no interference was observed between the cardiac pacemaker and deep brain stimulator when the cardiac pacemaker was interrogated and the intracardiac electrocardiograms were reviewed. The 24-hour rhythm follow-up in the pacemaker records revealed no sign of interference during this 6 month period.

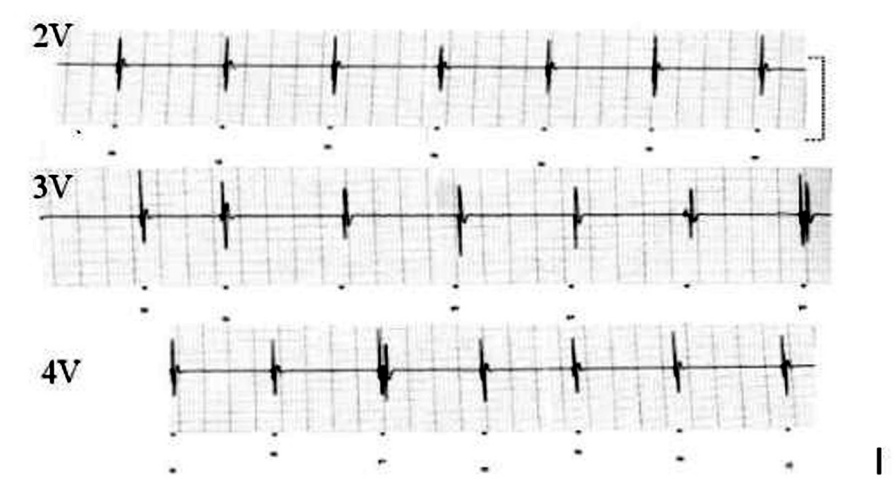

Figure 3. Intracardiac electrocardiogram detected by pacemaker lead showing that there was no electromagnetic interference at different voltage levels of the deep brain stimulator (from lowest to highest voltage). 


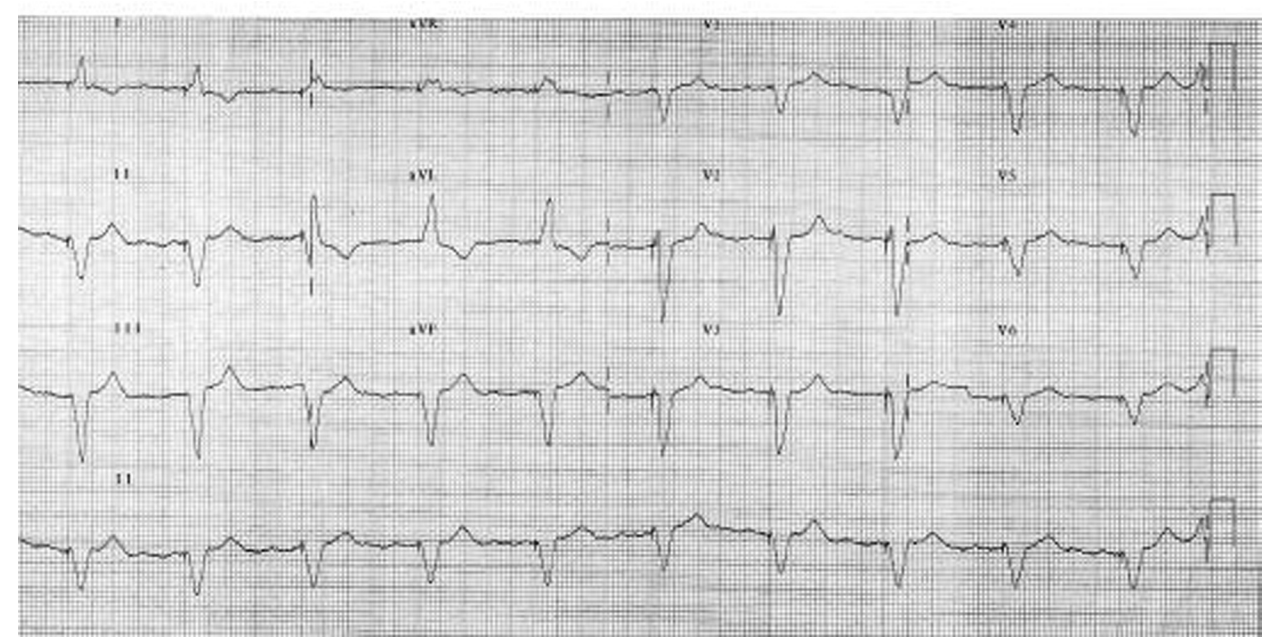

Figure 4. ECG showing regular pacemaker rhythm.

\section{DISCUSSION}

The past decade has seen an increase in the use of implantable chips and biosensors for the treatment of certain diseases. Among them, implantable cardiac pacemakers and defibrillators play a significant role and are commonly used therapeutic modalities for certain cardiac arrhythmias. ${ }^{1)}$ Deep brain stimulation at high frequency was first used in 1997 to replace thalamotomy in treating the characteristic tremor of Parkinson's disease, and has subsequently been applied to the pallidum and the subthalamic nucleus. Bilateral electrical stimulation of the subthalamic nucleus is now being used with increasing frequency in the treatment of selected patients with disabling, drug-refractory essential tremor or advanced Parkinson's disease. ${ }^{2)}$

There are concerns about the potential electrical interaction and interference between these 2 devices (or with device programming) in the same patient. Unfortunately, reports on this issue are limited. A small number of case reports, either about the implantation of bilateral deep brain stimulators in patients with Parkinson's disease and preexisting cardiac pacemakers, ${ }^{5,6}$ or the implantation of a cardiac pacemaker/ICD in a patient with preexisting deep brain stimulators ${ }^{7-10}$ ) are present in the literature. Whether or not their implantations in the same patient may cause electromagnetic interference is controversial; it has been reported that ICDs and cardiac pacemakers interfere with cellular telephones ${ }^{11)}$ and antitheft surveillance devices. ${ }^{12)}$ Stored electrocardiograms of ICDs showed that in some 
instances, electromagnetic interference was interpreted as ventricular fibrillation and this might even lead to inappropriate shocks. ${ }^{13)}$ Apart from the device-device interactions, Kaufmann, et $a l^{14)}$ reported that stimulation of the subthalamic nucleus increased heart rate in patients with Parkinson's disease.

Due to the probability of complications and possible interference between the devices, patients should be informed prior to performing a procedure. However, cardiac pacemakers and ICD devices are life-saving and deep brain stimulators allow patients to carry on their daily activities. Thus, the use of these devices seems to be undeniable. Actually, in one report, when the patient was told that his deep brain stimulator had to be permanently deactivated, the patient declined saying that he would rather risk sudden cardiac death than resume life with a disabling tremor. ${ }^{7)}$

In most of the reports, ${ }^{5,7-9)}$ it was stated that testing during implantation showed that the neurostimulators did not affect the bipolar sensing of the ICD or pacemakers even when the stimulators were set to maximum limits. No interference was noted at any time during either the implantation or follow-up period, and both pacemakers and stimulators functioned well. Rosenow, et $a l^{8)}$ reported that after implantation of an ICD in a patient with a deep brain stimulator, testing of the devices over a wide range of settings revealed no interaction and the patient subsequently underwent multiple episodes of cardioversion with no evidence of adverse neurological effects, and interrogation of the DBS devices after cardioversions revealed no changes in stimulus parameters indicating that DBS systems might be safely retained in selected patients who required ICDs. On the other hand, only one of the reports stated that the ICD shock did affect the stimulators. ${ }^{9)}$ However, Capelle, et $a l^{6}$ studied 6 patients with cardiac pacemakers who required deep brain stimulators for their movement disorders refractory to treatment. They reported that there were no adverse events during surgery and no interference was detected between the 2 systems during 2 years of follow-up of the patients, indicating that on the contrary to previous approaches, cardiac pacemakers should not be viewed as a general contraindication for deep brain stimulation in patients with movement disorders.

During implantation of the cardiac pacemakers or ICD, certain parameters should be carefully monitored. Because of concern about possible interaction between these devices, deep brain stimulation systems are routinely disconnected before defibrillators are implanted. ${ }^{8)}$ The deep brain stimulator pulse generator and the cardiac pacemaker should be placed on opposite sides of the chest. The distance between the devices should be appropriate to facilitate interrogation and programming of one device without affecting the other device. Patients are subject to electromagnetic interference during an electrosurgical procedure; in one 
report, to minimize electromagnetic interference, homeostasis was obtained with the aid of a battery-operated heat-generating handheld electrocautery device. ${ }^{15}$ )

The choice of the ICD ventricular lead may also influence whether the device senses the deep brain stimulator. Single coil ICD leads have a true bipolar electrode at the tip, which facilitates better sensing at the endocardial interface. When a dual coil lead is used, the distal coil and tip of the lead comprise the bipolar electrode, increasing the surface area of detection and therefore increasing the likelihood of inappropriate sensing. ${ }^{7)}$

Another source of complication could be the telemetric programmers of the ICD and deep brain stimulators. The deep brain stimulator pulse generators can be activated or deactivated with a telemetric programmer or with a special magnet like pacemakers and ICD. The telemetric ICD programmer is able to deactivate the pulse generator of the deep brain stimulator leading to recurrence of tremor. ${ }^{7)}$ This is highly important as tremor might cause a phenomenon called pseudoventricular tachycardia, which can be misdiagnosed by the ICD leading to inappropriate shock. However, the deep brain stimulator programmer or magnet that is used by the patient to activate or deactivate the pulse generator cannot damage an ICD if unintentionally placed directly over it; but will, depending on the company and model, cause the ICD to suspend detection while a magnetic field is present or deactivate the ICD. ${ }^{7)}$ False programming by unintended erroneous placement of the respective programmers over the wrong pulse generator is not possible because these programmers identify the matching device by a digital code. However, controlling the devices is advised after reprogramming.

Conclusion: Deep brain stimulation has become an important modality in the treatment of refractory Parkinson's disease. In patients with comorbid arrhythmias requiring cardiac pacemakers, there are concerns over a possible electrical interaction between the devices. Our case suggests that treatment with concomitant deep brain stimulators and cardiac pacemakers - defibrillator devices may be safe and effective and that the removal of existing devices may be unnecessary. However, patients undergoing implantation and programming of these devices should be informed of the possible interactions.

\section{REFERENCES}

1. Gregoratos G, Abrams J, Epstein AE, et al; American College of Cardiology/American Heart Association Task Force on Practice Guidelines/North American Society for Pacing and Electrophysiology Committee to Update the 1998 Pacemaker Guidelines. ACC/AHA/NASPE 2002 guideline update for implantation of cardiac pacemakers and antiarrhythmia devices: summary article: a report of the American College of Cardiology/American Heart Association Task Force on Practice Guidelines (ACC/AHA/NASPE Committee to Update the 1998 Pacemaker Guidelines). Circulation 2002; 106: 2145-61. 
2. Benabid AL, Pollak P, Gervason C, et al. Long-term suppression of tremor by chronic stimulation of the ventral intermediate thalamic nucleus. Lancet 1991; 337: 403-6.

3. Benabid AL, Pollak P, Gao D, et al. Chronic electrical stimulation of the ventralis intermedius nucleus of the thalamus as a treatment of movement disorders. J Neurosurg 1996; 84: 203-14.

4. Pinski SL, Fahy GJ. Implantable cardioverter-defibrillators. Am J Med 1999; 106: 446-58. (Review)

5. Senatus PB, McClelland S 3rd, Ferris AD, et al. Implantation of bilateral deep brain stimulators in patients with Parkinson disease and preexisting cardiac pacemakers. Report of two cases. J Neurosurg 2004; 101: 1073-7.

6. Capelle HH, Simpson RK Jr, Kronenbuerger M, Michaelsen J, Tronnier V, Krauss JK. Long-term deep brain stimulation in elderly patients with cardiac pacemakers. J Neurosurg 2005; 102: 53-9.

7. Obwegeser AA, Uitti RJ, Turk MF, et al. Simultaneous thalamic deep brain stimulation and implantable cardioverter-defibrillator. Mayo Clin Proc 2001; 76: 87-9.

8. Rosenow JM, Tarkin H, Zias E, Sorbera C, Mogilner A. Simultaneous use of bilateral subthalamic nucleus stimulators and an implantable cardiac defibrillator. Case report. J Neurosurg 2003; 99: 167-9.

9. Tavernier R, Fonteyne W, Vandewalle V, de Sutter J, Gevaert S. Use of an implantable cardioverter defibrillator in a patient with two implanted neurostimulators for severe Parkinson's disease. Pacing Clin Electrophysiol 2000; 23: 1057-9.

10. Kazatsker M, Kusniek J, Hasdai D, Battler A, Birnbaum Y. Two pacemakers in one patient: a stimulating case. J Cardiovasc Electrophysiol 2002; 13: 522.

11. Hayes DL, Wang PJ, Reynolds DW, et al. Interference with cardiac pacemakers by cellular telephones. N Engl J Med 1997; 336: 1473-9.

12. Santucci PA, Haw J, Trohman RG, Pinski SL. Interference with an implantable defibrillator by an electronic antitheft-surveillance device. N Engl J Med 1998; 339: 1371-4.

13. Llinas R, Henderson GV. Images in clinical medicine. Tremor as a cause of pseudo-ventricular tachycardia. $\mathrm{N}$ Engl J Med 1999; 341: 1275.

14. Kaufmann H, Bhattacharya KF, Voustianiouk A, Gracies JM. Stimulation of the subthalamic nucleus increases heart rate in patients with Parkinson disease. Neurology 2002; 59: 1657-8.

15. Martinelli PT, Schulze KE, Nelson BR. Mohs micrographic surgery in a patient with a deep brain stimulator: a review of the literature on implantable electrical devices. Dermatol Surg 2004; 30: 1021-30. (Review) 